\title{
Tratamiento contable de las stock options según la normativa contable internacional
}

\section{Elena Merino Madrid}

Profesora Asociada, Facultad de Ciencias Jurídicas y Sociales. Universidad de Castilla-La Mancha.Elena.Merino@uclm.es

Regino Banegas Ochovo Catedrático, Facultad de Ciencias Sociales, Universidad de Castilla-La Mancha. Regino. Banegas@uclm.es

\section{Jesús Fernando Santos}

Peñalver

Catedrático, Facultad de Derecho y Ciencias Sociales, Universidad de Castilla-La Mancha.Jesus.Santos@uclm.es

\section{Resumen}

La retribución que hacen las empresas a sus directivos y empleados mediante la entrega de opciones sobre acciones (stock options) se ha convertido en una práctica habitual en muchos países del mundo. Hasta fechas recientes no existía un consenso sobre el tratamiento contable que se debía dar a este tipo de transacciones; sin embargo, en la actualidad parece que tal consenso se ha alcanzado al exigir tanto el International Accounting Standards Board (IASB) como el Financial Accounting Standards Board (FASB), en la International Financial Reporting Standard, IFRS 2 (NIIF 2, Norma Internacional de Información Financiera) y Statement of Financial Accounting Standards (SFAS) $123(\mathrm{R})$ respectivamente, que se reconozca la remuneración basada en la entrega de opciones sobre acciones como un gasto en los estados financieros. El objetivo de este trabajo es analizar el tratamiento contable aplicable de acuerdo con la normativa contable internacional o de información financiera (NIC/NIIF).

Palabras clave: normativa contable internacional, NIIF 2, planes de opciones sobre acciones, stock options. 


\title{
Accounting for stock options under the international accounting standard
}

\begin{abstract}
The stock options grants by companies to their executives and employees have become a common practice in many countries worldwide. Until recently, there was no consensus on the accounting treatment to be applied to such transactions. But now it seems that consensus has been reached, through the requirements of both the IASB and the FASB, under IFRS 2 and SFAS 123 (R) respectively, to recognize stock option grants as an expense in their financial statements. It is the aim of this paper to analyze the accounting treatment applicable under international accounting standards (IAS/IFRS).
\end{abstract}

Keywords: International Accounting Standards, IFRS 2, share-based payment, stock options.

\section{Introducción}

Las opciones sobre acciones (stock options) surgen como un sistema novedoso de retribución variable a largo plazo, la cual consiste en un contrato tipo call que otorga a los beneficiarios el derecho, que no la obligación, de comprar normalmente en una fecha establecida, en un número determinado de acciones de la empresa oferente del plan o de alguna de las empresas del grupo a un precio prefijado. Al tratarse de un plan retributivo, es norma generalizada la exención del pago de la prima, pues la contraprestación del plan es el propio trabajo realizado por los beneficiarios. En ocasiones, el ejercicio de las opciones está supeditado al cumplimiento de objetivos empresariales específicos, tales como alcanzar determinadas ventas o rendimientos. Finalmente, en el momento de ejecución de las opciones, el beneficio vendrá dado por la diferencia entre el precio de ejercicio y el valor de las acciones en el mercado. Este beneficio, en principio, es ilimitado al estar en función de cómo crezca el valor de las acciones sin que exista ningún tipo de riesgo para el beneficiario.

El procedimiento habitual de liquidación de los planes retributivos de opciones sobre acciones, y que diferencia a esta figura de otras, consiste en la compra de acciones por parte de los beneficiarios, si así lo deciden, cuando llegue el periodo de ejercicio. En ocasiones, sin embargo, los planes incluyen una cláusula que faculta a la empresa o al empleado a decidir si la liquidación, a la fecha de ejercicio, se realizará mediante la compra de las acciones o mediante la entrega de un importe en metálico. En este último caso no estaríamos ante un plan de opciones sobre 
acciones sino ante otra figura retributiva denominada bonus diferenciales o stock appreciation rights.

Desde la incorporación de las stock options en los planes retributivos del mundo empresarial, se ha planteado la pregunta: $¿$ deben considerarse como un gasto para la empresa? La respuesta no resulta fácil porque los diversos organismos reguladores, durante mucho tiempo, no han exigido a las empresas que los reconozcan en sus estados de resultados, conformándose con su inclusión en las notas complementarias. Sin embargo, siempre ha sido un tema recurrente, aunque también han sido muchos los argumentos esgrimidos en contra de la consideración de la retribución mediante la entrega de stock options como un gasto, lo que, sin duda, ha dilatado el proceso de aprobación de una norma al respecto.

En el nivel internacional la Norma Internacional de Información Financiera (NIIF) $2^{1}$ y en Estados Unidos de Norteamérica la Statement of Financial Accounting Standards (SFAS) 123 (R) han puesto un punto final a este debate al exigir que se registren en las cuentas de resultados los gastos ocasionados por la remuneración basada en la entrega de stock options a los consejeros, directivos y trabajadores.

El presente trabajo analiza la problemática contable de las opciones sobre acciones, regulada en la normativa contable internacional, considerando los siguientes aspectos: el valor razonable como método finalmente elegido para llevar a cabo la valoración del gasto, la fecha en que debe determinarse dicho valor y los factores básicos a tener en cuenta, con especial referencia a la volatilidad y a los dividendos. Además, se destacan otros factores por considerar para el cálculo del valor razonable tales como el ejercicio anticipado o las condiciones por cumplir para llevar a cabo el ejercicio, así como el gasto total por reconocer y el periodo de contabilización de dicho gasto.

\footnotetext{
${ }^{1}$ Esta norma junto con el resto de normativa internacional son de obligada aplicación: a) para todos los países miembros de la Unión Europea en la elaboración de las cuentas consolidadas de los grupos que coticen en cualquiera de sus mercados de valores (Reglamento 1606/2002 del Parlamento Europeo) y b) para las empresas cotizadas nacionales en más de 40 países en todo el mundo. Además, el uso de la normativa internacional también está permitida en otros tantos países. Finalmente, en aquellos países donde el uso de la normativa internacional no está permitida se está trabajando para conseguir una armonización entre sus propias normas y aquéllas.
} 


\section{Método de valoración de las stock options como gasto: el valor razonable}

Cuando se adquieren bienes o se reciben servicios a cambio de la entrega de instrumentos de patrimonio neto la NIIF 2 establece, como regla general, que se utilice el valor razonable de dichos bienes o servicios. Sin embargo, para las transacciones realizadas con los empleados (consejeros, directivos y trabajadores) el criterio de valoración utilizado debe ser el valor razonable de los instrumentos de patrimonio otorgados en lugar del criterio general (ver cuadro 1). Ello se basa en la dificultad de valorar el servicio recibido porque, normalmente, los instrumentos de patrimonio se conceden a los empleados como una parte de su remuneración, junto con el sueldo en efectivo y otras prestaciones. Esto hace difícil valorar directamente los servicios recibidos por cada componente concreto que forme parte del conjunto de remuneraciones de los empleados.

También se contempla la posibilidad de dificultades a la hora de estimar el servicio recibido de los beneficiarios si las opciones sobre acciones se conceden no como parte del salario básico, sino como forma de bonificación, bien por permanecer en la empresa durante un determinado periodo o bien como recompensa por los esfuerzos realizados en la mejora del rendimiento de la misma.

\section{Cuadro 1}

Criterios de valoración de las operaciones de entrega de instrumentos de patrimonio neto según la NIIF 2

\begin{tabular}{lll}
\hline \multicolumn{1}{c}{ Tipo de operación } & \multicolumn{1}{c}{ Regla general } & \multicolumn{1}{c}{ Otros métodos } \\
\hline $\begin{array}{l}\text { Entrega de instrumentos } \\
\text { de neto a empleados }\end{array}$ & $\begin{array}{l}\text { Valor razonable de los } \\
\text { instrumentos de neto }\end{array}$ & $\begin{array}{l}\text { Valor intrínseco (en las raras } \\
\text { ocasiones en que el valor } \\
\text { razonable de los instrumentos de } \\
\text { neto no se pueda estimar) }\end{array}$ \\
\hline $\begin{array}{l}\text { Entrega de instrumentos de } \\
\text { neto a sujetos distintos a los } \\
\text { empleados }\end{array}$ & $\begin{array}{l}\text { Valor razonable de los bienes } \\
\text { o servicios }\end{array}$ & $\begin{array}{l}\text { Valor razonable de los } \\
\text { instrumentos de neto (si no } \\
\text { puede estimar el valor razonable } \\
\text { de los bienes o servicios) }\end{array}$ \\
\hline
\end{tabular}

Fuente: Elaboración propia

Cuando el valor razonable de los instrumentos de patrimonio neto no se pueda estimar con fiabilidad se podrá utilizar el valor intrínseco entendido como "la diferencia entre el valor razonable de las acciones que la otra parte tiene derecho 
(condicional o incondicional) a suscribir, o que tiene derecho a recibir y el precio (si existiese) que la otra parte está (o estará) obligada a pagar por esas acciones" (NIIF 2, 2005: apéndice A). No obstante, la normativa reconoce, como raras excepciones, las ocasiones en las que no se pueda estimar el valor razonable de los instrumentos de patrimonio neto entregados.

Cuando las transacciones basadas en acciones ofrezcan la posibilidad de elegir la liquidación a través de la entrega de instrumentos de patrimonio o en metálico, la normativa contable internacional indica que deberán aplicarse una serie de criterios en función de que dicha elección pueda realizarla el empleado o la empresa.

\section{Fecha y factores para la determinación del valor razonable de los instrumen- tos de patrimonio neto}

\section{Fecha para la determinación del valor razonable}

Según la NIIF 2 (2005), y para el caso de las transacciones con empleados, la fecha apropiada para la determinación del valor razonable es la que se conoce como de concesión (grant date), entendida ésta como "la fecha en la que la entidad y un tercero alcanzan un acuerdo de pagos basados en acciones, que se produce cuando la entidad y la otra parte llegan a un entendimiento compartido sobre los plazos y condiciones del acuerdo" (NIIF 2, 2005: apéndice A).

En el caso de que el valor razonable no se pueda estimar con fiabilidad se utilizará el valor intrínseco calculándose, inicialmente, en la fecha en que la empresa obtenga los bienes o la otra parte preste los servicios.

\section{Cuadro 2}

Fecha de valoración del valor razonable según NIIF 2

\begin{tabular}{ll}
\hline \multicolumn{1}{c}{ Tipo de operación } & \multicolumn{1}{c}{ Fecha de valoración } \\
\hline $\begin{array}{l}\text { Transacciones con empleados } \\
\text { (y terceros que prestan servicios similares) }\end{array}$ & Fecha de concesión \\
\hline Transacciones con sujetos distintos a los empleados & $\begin{array}{l}\text { Fecha en que se obtienen los bienes o la otra } \\
\text { parte presta los servicios }\end{array}$ \\
\hline
\end{tabular}

Fuente: Elaboración propia 
El requerimiento de la normativa contable, tanto en la NIIF 2 como en la SFAs 123 (R), de utilizar la fecha de concesión para la determinación del valor razonable del gasto de las stock options ha recibido gran cantidad de críticas (Niskanen, 2004: 1; Templin, 2005: 394), provocando que la doctrina venga proponiendo fechas alternativas. A estos efectos, la propia norma SFAs 123 (R) las recoge en su Apéndice B exponiendo los motivos por los que no se permite su utilización y las razones por las que finalmente la fecha de concesión es la más apropiada. Por su parte, la NIIF 2 también recoge algunas de estas razones en las Bases para las Conclusiones en los párrafos BC80 al BC105.

A pesar de las críticas, y considerando la entrega de opciones sobre acciones como un costo de compensación, éste debe ser calculado en el momento que se produce la concesión de las citadas opciones, fecha de concesión o gran date, sin ser posible utilizar otra fecha porque cualquiera sería posterior al momento en que la empresa incurre en el gasto como consecuencia de la aprobación de la entrega de las citadas opciones.

\section{Factores básicos para la determinación del valor razonable}

En términos similares al tratamiento contable recogido en otras normas internacionales, referentes al valor razonable, la NIIF 2 lo define, en líneas generales, como "el importe por el que un activo podría ser intercambiado, un pasivo, liquidado, o un instrumento de patrimonio concedido, entre partes interesadas y debidamente informadas, en una transacción realizada en condiciones de independencia mutua". (NIIF 2, 2005: apéndice A).

En principio, y con carácter general, para la determinación del valor razonable de los instrumentos de patrimonio concedidos se considerarán "los precios de mercado si estuvieran disponibles, teniendo en cuenta los plazos y condiciones sobre los que esos instrumentos de patrimonio fueron concedidos" (NIIF 2, 2005: párrafo 16). En particular, y para el caso de la concesión de opciones sobre acciones a los empleados, es posible que no estén disponibles los precios de mercado "ya que dichas opciones están sujetas a plazos y condiciones que no se aplican a las opciones cotizadas. Si no existieran opciones cotizadas con plazos y condiciones parecidos, el valor razonable de las opciones concedidas se estimará aplicando un modelo de valoración de opciones". (NIIF 2, 2005: párrafo B4). 
En este sentido, la NIIF 2 no define los modelos de valoración específicos utilizables, sino que se limita a describir los factores que, como mínimo, deberán considerarse al estimar el valor razonable de los pagos basados en acciones ${ }^{2}$. La técnica de valoración elegida, cualquiera que sea, deberá ser "coherente con los métodos de valoración generalmente aceptados para la fijación de precios de los instrumentos financieros, e incorporará todos los factores e hipótesis que sean conocidas, y que considerarían los partícipes en el mercado a la hora de fijar el precio" (NIIF 2, 2005: párrafo 17). En la línea de la normativa contable internacional, la SFAs 123 (R) (2004: párrafo A14) tampoco se decanta por un modelo de valoración de opciones sobre acciones en especial siempre y cuando el modelo elegido cumpla una serie de objetivos y requisitos.

El valor de la opción sobre acciones suele medirse aplicando modelos de valoración generalmente aceptados en los mercados financieros (ver cuadro 3), tales como la fórmula de Black-Scholes (1973) (que no incluye los dividendos) o la de Black-Scholes y Merton (que sí los incluye) o el modelo binomial de Cox-RossRubinstein (1979) (ver anexo).

\section{Cuadro 3 \\ Modelos de valoración de opciones de compra europeas}

\begin{tabular}{cl}
\hline \multicolumn{1}{c}{ Tipos } & \multicolumn{1}{c}{ Modelos desarrollados } \\
\hline \multirow{3}{*}{ Métodos analíticos } & $\begin{array}{l}\text { Fórmula de Black-Scholes (1973) que parte de la hipótesis de que no se reparten } \\
\text { dividendos a lo largo de la vida de la opción }\end{array}$ \\
\cline { 2 - 2 } & $\begin{array}{l}\text { Fórmula de Black-Scholes y Merton (1973) que parte de la hipótesis de la existencia } \\
\text { de reparto de dividendos }\end{array}$ \\
\hline \multirow{2}{*}{$\begin{array}{c}\text { Métodos } \\
\text { numéricos }\end{array}$} & Método de simulación de Montecarlo desarrollado por Boyle (1977) \\
\cline { 2 - 2 } & Método de diferencias finitas desarrollado por Brennan y Schwartz (1977 y 1978) \\
\cline { 2 - 2 } & Método binomial desarrollado por Cox, Ross y Rubinstein (1979) \\
\cline { 2 - 2 } & Método trinomial desarrollado por Boyle (1986) \\
\hline
\end{tabular}

Fuente: Elaboración propia

\footnotetext{
${ }^{2}$ Esto se ha hecho así deliberadamente tal y como recoge el Considerando (4) del Reglamento (CE) no 211/2005 "para no obstaculizar el desarrollo de técnicas de medición adecuadas, todavía inexistentes, de todo tipo de pagos basados en acciones (por ejemplo, opciones de compra de acciones de los empleados a largo plazo no negociables)". A continuación señala que, seguramente, en el futuro se desarrollarán métodos adecuados para realizar la estimación de este tipo de instrumentos retributivos, teniendo en cuenta sus características, en especial "las empresas de reciente cotización y las empresas que carecen de experiencia suficiente pueden tener dificultades para estimar el precio futuro de las acciones".
} 
Para superar las deficiencias que presentan estos modelos de valoración de las opciones sobre acciones entregadas a los empleados se propone incluir variaciones a éstos; por ejemplo, considerar el ejercicio anticipado, en cuyo caso se puede utilizar la vida que se espera que tenga la opción en lugar de su vida total.

A pesar de los esfuerzos de los expertos por desarrollar un modelo de valoración de uso exclusivo para las opciones sobre acciones entregadas a los empleados (Lambert et al., 1991; Huddart, 1994; Kulatilaka y Marcus, 1994; Rubinstein, 1994; Carpenter, 1998; Carr y Linetsky, 2000; Raupach, 2003; Hull y White, 2004; Bulow y Shoven, 2005; Finnerty, 2005; etc.), que contemple sus características específicas, ninguno de los desarrollados hasta ahora ha merecido la atención de los organismos contables internacionales. De ahí que tanto el FASB como el IASB no recojan un modelo de valoración concreto que deban aplicar las empresas a la hora de realizar el cálculo del valor razonable de las opciones sobre acciones, dejando dicha elección a la discreción de cada una de ellas. En cualquier caso, todos los modelos de valoración de opciones sobre acciones deben contemplar, como mínimo, los factores siguientes (NIIF 2, 2005: párrafo B6):

a) El precio de ejercicio. Es el importe que el beneficiario deberá pagar cuando llegue el periodo de ejercicio, es decir, si finalmente aquél decide ejercer su opción de compra. El precio de ejercicio afecta de forma inversamente proporcional al valor de la opción (Córdoba, 2001: 35). Así, el valor de una opción será mayor cuanto menor sea el precio de ejercicio (Lamothe, 1993: 47) porque existirá una mayor probabilidad de que el precio de mercado de la acción acabe superándolo (Díez y Mascareñas, 1991: 101).

b) Vida de la opción. Una opción, a mayor tiempo, tendrá mayor valor (Lamothe, 1993: 46), dado que, razonablemente, cuanto mayor sea la vida de la opción mayor probabilidad existe de que suba o baje el valor del activo subyacente y, por tanto, de que se puedan ejercitar las opciones (Córdoba, 2001: 35; Borrego y García, 2002: 260). Si bien los modelos tradicionales de valoración de opciones consideran como variable la vida total de la opción (desde la fecha de emisión de la opción hasta la fecha de expiración o caducidad de la misma), la norma internacional (NIIF 2, 2005: párrafo B17) recomienda la utilización del periodo de vida esperado de la opción en lugar del periodo máximo. ${ }^{3}$

\footnotetext{
${ }^{3}$ Ver el apartado Ejercicio anticipado de las opciones.
} 
c) Precio del activo subyacente. El valor de la opción es directamente proporcional al valor del activo subyacente (Córdoba, 2001: 35), por cuya razón las alzas en los precios del subyacente provocan subidas del valor de la opción (Díez y Mascareñas, 1991: 99; Lamothe, 1993: 41).

d) Volatilidad. Constituye el factor clave para la valoración de las opciones sobre acciones, puesto que es el único factor que no se conoce y que no se puede determinar a través del uso de modelo o fórmula alguno (Mock, 2005: 365). La volatilidad se refiere al posible rango de variaciones de los precios del activo subyacente (Lamothe, 1993: 43). La medida de la volatilidad usada en los modelos de valoración de opciones es la desviación típica ${ }^{4}$ anualizada de las tasas de rendimiento sobre las acciones a lo largo de un periodo, utilizando para su cálculo la capitalización continua. Para la estimación de la volatilidad esperada deben incluirse una serie de factores recogidos por la normativa contable internacional (NIIF 2, 2005: párrafo B25) (ver cuadro 4).

\section{Cuadro 4}

\section{Factores por considerar en la estimación de la volatilidad esperada según la NIIF 2}
Tipo de
entidad
Factores por considerar

\begin{tabular}{ll} 
& - Volatilidad implícita de las opciones sobre acciones (o de otros instrumentos similares) \\
Entidades & - Volatilidad histórica del precio de la acción en el periodo más reciente \\
cotizadas & - Extensión temporal del periodo durante el que las acciones de la entidad han cotizado \\
& - La tendencia de la volatilidad a revertir a su media \\
& - El uso de intervalos regulares y adecuados para las observaciones de los precios \\
\hline & - Si la sociedad ha establecido un mercado interno de sus acciones, puede utilizar la volatilidad \\
Entidades & del precio de esas acciones \\
no cotizadas & - Podría utilizar la volatilidad histórica o implícita de entidades similares cotizadas \\
& - Si la entidad hubiera utilizado otra metodología para valorar sus acciones, podrá realizar una \\
& estimación de la volatilidad esperada con base en esa metodología
\end{tabular}

Fuente: Elaboración propia

\footnotetext{
${ }^{4}$ Los modelos de valoración de opciones asumen la hipótesis de un mercado eficiente para el subyacente; es decir que los precios del subyacente incorporan automáticamente toda la información relevante sobre dicho subyacente. Si el mercado es eficiente, la variación de los precios será totalmente aleatoria y la distribución estadística de los precios se aproximará a una distribución normal. En una distribución normal, el nivel de dispersión de los valores posibles de la variable lo podemos medir por la varianza o desviación típica (Lamothe, 1993: 105).
} 
e) Dividendos. Los dividendos afectan al precio de la opción porque cuando las empresas los pagan desciende la cotización bursátil, por cuyo motivo el valor de la opción disminuiría. El considerar o no los dividendos esperados para determinar el valor razonable de las opciones dependerá del otorgamiento a los "beneficiarios del plan de opciones" el derecho a recibir éstos o una retribución equivalente a los mismos:

- Si se ha concedido a los "beneficiarios de las opciones" el derecho a percibir dividendos entre la fecha de la concesión y la de ejercicio "las opciones concedidas deberán valorarse como si no se fuesen a pagar dividendos sobre las acciones subyacentes; es decir, el valor de la variable representativa de los dividendos debería ser cero" (NIIF 2, 2005: párrafo B32).

- $\mathrm{Si}$, por el contrario, los empleados no tienen derecho a recibir dividendos o retribuciones equivalentes a éstos antes del ejercicio de la opción, la valoración en la fecha de concesión deberá considerar los dividendos esperados. Esto significa que al estimar el valor razonable de una concesión de opciones los dividendos esperados deberán ser incluidos en la aplicación del modelo de valoración de opciones (NIIF 2, 2005: párrafo B34).

\section{Cuadro 5 \\ Inclusión de los dividendos esperados en los modelos de valoración de opciones según la NIIF 2}

\begin{tabular}{ll}
\hline \multicolumn{1}{c}{ Situación } & \multicolumn{1}{c}{ Variable de los dividendos } \\
\hline $\begin{array}{l}\text { Si los beneficiarios de opciones reciben } \\
\text { dividendos }\end{array}$ & $\begin{array}{l}\text { El valor de la variable representativa de los dividendos en el } \\
\text { modelo de valoración de opciones debería ser cero }\end{array}$ \\
\hline $\begin{array}{l}\text { Si los beneficiarios de opciones no reciben } \\
\text { dividendos }\end{array}$ & $\begin{array}{l}\text { Los dividendos estimados deberán ser incluidos en la aplicación } \\
\text { del modelo de valoración de opciones }\end{array}$ \\
\hline
\end{tabular}

Fuente: Elaboración propia

Con carácter general, los modelos de valoración de opciones sobre acciones exigen la utilización de una tasa de rentabilidad esperada por dividendos. Sin embargo, también podría usarse un importe de dividendo esperado en lugar de una tasa de rentabilidad, en cuyo caso deberá considerar su patrón histórico de incremento de los citados dividendos (NIIF 2, 2005: párrafo B35). 


\section{Cuadro 6}

Valoración de los dividendos esperados según la NIIF 2

\begin{tabular}{ll}
\hline \multicolumn{1}{c}{ Tipo de entidad } & \multicolumn{1}{c}{ Valoración de los dividendos esperados } \\
\hline Entidades con historial & $\begin{array}{l}\text { - Rentabilidad esperada de los dividendos } \\
\text { - Importe de los dividendos esperados }\end{array}$ \\
\hline Entidades emergentes & $\begin{array}{l}\text { - Pueden utilizar una media entre sus rentabilidades } \\
\text { pasadas por dividendos (cero) y la rentabilidad media por } \\
\text { dividendos de un grupo similar comparable }\end{array}$ \\
\hline
\end{tabular}

Fuente: Elaboración propia

f) Tasa de interés libre de riesgo. Con frecuencia la tasa de interés libre de riesgo es la rentabilidad implícita, actualmente disponible para las emisiones cupón cero de los organismos públicos de aquellos países en cuya moneda se expresa el precio de ejercicio con un plazo restante igual al esperado de la opción que va a ser valorada (basado en la vida contractual restante de la vida de la opción y considerando los efectos de un eventual ejercicio anticipado esperado). Sin embargo, podría ser necesario emplear un sustituto adecuado en determinadas ocasiones (NIIF 2, 2005: párrafo B37) (ver cuadro 7).

\section{Cuadro 7}

Valoración de la tasa de interés libre de riesgo según la NIIF 2

\begin{tabular}{ll}
\hline \multicolumn{1}{c}{ Criterios } & \multicolumn{1}{c}{ Valoración de la tasa de interés } \\
\hline Criterio general & Rentabilidad implícita para emisiones cupón cero de los organismos públicos \\
\hline & Se utilizará un criterio sustituto al general si se da alguna de las siguientes situaciones: \\
1) Si no existiesen las citadas emisiones cupón cero
\end{tabular}

Fuente: Elaboración propia

Otros factores por considerar para determinar el valor razonable

Además de los factores antes citados, y que deben ser la base a la hora del cálculo del valor razonable, si existe algún otro factor que un "sujeto interesado y debida- 
mente informado" podría considerar al establecer el precio, también se tendrá en cuenta. Sin embargo, en ningún caso se contemplarán los factores que afectan sólo desde la perspectiva individual del empleado debido a que, en estos casos, dichos factores se consideran como irrelevantes para la estimación del precio.

La normativa contable internacional no hace una enumeración de los factores que deben observarse y aplicarse; no obstante, sí presta especial atención al ejercicio anticipado, que puede darse en determinados circunstancias y a las condiciones que, en muchas ocasiones, deben cumplir los beneficiarios para poder adquirir los derechos de compra sobre las acciones.

\section{Ejercicio anticipado de las opciones}

En ocasiones, los beneficiarios de un plan retributivo de opciones sobre acciones pueden ejercitar las opciones anticipadamente por diversos motivos, entre los que se encuentran los siguientes:

- Es el único modo de liquidar su posición, puesto que las opciones sobre acciones son, normalmente, intransmisibles. Esto puede dar lugar a que los beneficiarios las ejerciten anticipadamente, con frecuencia, nada más empezar el periodo de ejercicio.

- En los supuestos casos de jubilación, incapacidad, terminación del contrato o despido improcedente del beneficiario, a pesar de cesar su prestación de servicios, normalmente se permite el ejercicio anticipado de las opciones que se hayan consolidado hasta ese momento. En el resto de casos, y esencialmente si el empleado cesa sus servicios de forma voluntaria, no se permite el ejercicio anticipado, sino que simplemente se pierden las opciones.

- Existen otras situaciones que pueden conducir a que el beneficiario pretenda ejercitar las opciones de forma anticipada como, por ejemplo, el conocimiento de que la empresa va a entregar dividendos adicionales a los accionistas o ante información adversa sobre las perspectivas de futuro de la empresa. En estos casos, los beneficiarios podrían estar interesados en ejercitar anticipadamente las opciones (Maris et al., 2003: 671).

La normativa contable internacional indica que el modelo de valoración que se utilice para la determinación del valor razonable considere este factor de ejercicio anticipado, pues, en caso contrario, tal y como ha venido recogiendo la doctrina 
americana (Kulatilaka y Marcus, 1994: 47; Harter y Harikumar, 2002: 1008; Bettis et al., 2003: 37), el valor obtenido a través de los modelos tradicionales será superior al valor real de las opciones. Asimismo, se manifiesta que los medios para considerar los efectos del ejercicio anticipado esperado dependen del tipo de modelo de valoración que se aplique.

El ejercicio anticipado puede contemplarse en el modelo de valoración empleando una estimación de la vida esperada de la opción, que se define como el periodo desde la fecha de la concesión hasta la fecha en la que se espera que la opción sea ejercitada. Esta estimación aparece de acuerdo con las recomendaciones de la doctrina (Strawser, 1993: 3; Hemmer et al., 1994: 24; Bodie et al., 2003: 88), como una variable del modelo de valoración de opciones.

La normativa contable internacional (NIIF 2, 2005: párrafo B18) señala varios criterios para estimar la vida esperada y diversos factores por considerar (ver cuadro 8).

\section{Cuadro 8}

\section{Valoración del ejercicio anticipado de las opciones según la NIIF 2}

\begin{tabular}{ll}
\hline \multicolumn{1}{c}{ Aspectos } & \multicolumn{1}{c}{ Criterios } \\
\hline Ejercicio anticipado & $\begin{array}{l}\text { Debe considerarse, para valorar las opciones sobre acciones entregadas a } \\
\text { los empleados, la vida esperada de la opción }\end{array}$ \\
\hline $\begin{array}{l}\text { Criterios para estimar la vida } \\
\text { esperada }\end{array}$ & $\begin{array}{l}\text { - Vida media ponderada esperada para todo el grupo de } \\
\text { empleados } \\
\text { - Vida media ponderado según los subgrupos de empleados } \\
\text { pertenecientes al grupo }\end{array}$ \\
\hline $\begin{array}{l}\text { Factores por tener en cuenta } \\
\text { para la estimación de la vida } \\
\text { esperada }\end{array}$ & $\begin{array}{l}\text { a) La duración del periodo de consolidación de los derechos } \\
\text { b) El precio de las acciones subyacentes } \\
\text { c) El nivel del empleado en la organización }\end{array}$ \\
\hline
\end{tabular}

Fuente: Elaboración propia

Condiciones por cumplir para el ejercicio de las opciones

La concesión de opciones sobre acciones generalmente está sujeta al cumplimiento de ciertas condiciones tales como: 1) que el empleado siga prestando sus servicios en la empresa, 2) que se alcance un determinado rendimiento o 3) que consiga aumentar el importe de los beneficios o el precio de las acciones. 
En virtud de la normativa contable internacional, estas condiciones, dependiendo de si están referidas al mercado o no, tendrán una influencia diferente sobre el valor razonable de las opciones.

Las condiciones no referidas al mercado (por ejemplo, que el trabajador permanezca durante un determinado periodo prestando sus servicios para poder ejercitar las opciones) no se tendrán en cuenta al estimar el valor razonable de las opciones sobre acciones. Sin embargo, dichas condiciones sí se consideran para estimar el número de opciones que se prevé que se vayan a devengar. Así, durante el periodo de exclusión (durante el cual deben cumplirse las condiciones exigidas) se reconocerá el importe de los bienes adquiridos y servicios recibidos, utilizando la mejor estimación del número de opciones esperadas que se ejerciten. Si las informaciones posteriores indicasen que el número de opciones difiere de la estimación inicial, se procederá a revisar la misma. Por último, en la fecha de devengo o consolidación, la empresa revisará la estimación con el fin de que sea igual al número de opciones que cumplen las condiciones.

\section{Cuadro 9 \\ Procedimiento para tener en cuenta las condiciones asociadas al ejercicio de las opciones}

\begin{tabular}{|c|c|c|}
\hline Fecha & $\begin{array}{c}\text { Condiciones referidas } \\
\text { al mercado }\end{array}$ & $\begin{array}{c}\text { Condiciones no referidas } \\
\text { al mercado }\end{array}$ \\
\hline Día de concesión & $\begin{array}{l}\text { El valor razonable incluirá las condiciones } \\
\text { referidas al mercado }\end{array}$ & $\begin{array}{l}\text { El valor razonable no incluirá las } \\
\text { condiciones no referidas al mercado }\end{array}$ \\
\hline $\begin{array}{l}\text { Estados financieros } \\
\text { intermedios (entre la } \\
\text { fecha de concesión y } \\
\text { fecha de ejercicio) }\end{array}$ & $\begin{array}{l}\text { Gasto acumulado en esta fecha equivaldrá } \\
\text { al resultado de multiplicar la estimación } \\
\text { inicial de opciones y el valor razonable } \\
\text { inicial }\end{array}$ & $\begin{array}{l}\text { Gasto acumulado equivaldráal resultado } \\
\text { de multiplicar la última estimación de } \\
\text { opciones que se ejercitarán y el valor } \\
\text { razonable inicial }\end{array}$ \\
\hline $\begin{array}{l}\text { Final del periodo } \\
\text { del cumplimiento de } \\
\text { condiciones }\end{array}$ & $\begin{array}{l}\text { Gasto acumulado en esta fecha equivaldrá } \\
\text { al resultado de multiplicar la estimación } \\
\text { inicial de opciones y el valor razonable } \\
\text { inicial }\end{array}$ & $\begin{array}{l}\text { Gasto acumulado equivaldrá al } \\
\text { resultado de multiplicar las opciones } \\
\text { que efectivamente se ejerciten y el valor } \\
\text { razonable inicial }\end{array}$ \\
\hline
\end{tabular}

Fuente: Elaboración propia 
Por el contrario, aquellas condiciones exigidas que estén referidas al mercado (por ejemplo, un precio objetivo de la acción al que esté condicionado el ejercicio de la opción) afectarán a la estimación del valor razonable. Por ello, la empresa reconocerá los bienes adquiridos y servicios recibidos de la otra parte que satisfagan el resto de condiciones, independientemente de que se cumpla la condición relativa al mercado, sin que en ningún momento posterior pueda realizarse algún tipo de revisión.

\section{Reconocimiento del gasto}

El gasto por reconocer se calculará multiplicando el valor de las opciones sobre acciones que se haya obtenido, aplicando el valor razonable (o en su defecto el valor intrínseco), por el número de opciones estimadas que vayan a ejercerse.

El valor razonable, calculado para las opciones en la fecha de concesión, no se revisará en fechas posteriores a pesar de que el valor de la opción puede variar a lo largo del periodo dado, que se extiende desde la fecha de la concesión hasta la del ejercicio.

Para la estimación del número de opciones sobre acciones, deben observarse las condiciones exigidas para su ejercicio, estén referidas o no al mercado. La estimación realizada del número de opciones se revisará siempre que sea necesario y "la información posterior indique que el número de instrumentos de patrimonio que se espere consolidar difiera de las estimaciones previas" (NIIF 2, 2005: párrafo 20). Asimismo, se llevará a cabo una revisión en la fecha límite del cumplimiento de las condiciones exigidas a los beneficiarios (fecha de irrevocabilidad) para que "sea igual al número de instrumentos de patrimonio que finalmente cumplirán las condiciones para la irrevocabilidad” (NIIF 2, 2005: párrafo 20).

Una vez que la empresa haya reconocido los bienes adquiridos y servicios recibidos, como gasto, y el correspondiente incremento en el patrimonio neto, ésta no realizará, tras la fecha de irrevocabilidad, ajustes adicionales a dicho patrimonio neto. A título ilustrativo se puede señalar que la empresa no revertirá posteriormente el importe reconocido por los servicios recibidos de un empleado si las opciones no se llegan a ejercitar. Sin embargo, este requerimiento no impide que la empresa reconozca una transferencia dentro del patrimonio neto desde un componente a otro (NIIF 2, 2005: párrafo 23). 


\section{Periodo en que debe ser contabilizado el gasto}

La normativa contable internacional establece, como regla general, que las opciones sobre acciones otorgadas se reconozcan como gasto "en el momento de la obtención de dichos bienes o cuando dichos servicios sean recibidos" (NIIF 2, 2005: párrafo 7).

Ahora bien, la norma distingue tres situaciones diferentes, que pueden producirse dependiendo de las condiciones que se establezcan en el contrato (NIIF 2, 2005: párrafos 14 y 15):

- Que el derecho se consolide, inmediatamente, en el momento de la concesión.

- Que los planes especifiquen un periodo determinado para la consolidación de los derechos del empleado, no pudiéndose ejercitar la opción durante este periodo (periodo de exclusión).

- Que el periodo de exclusión (o de consolidación de los derechos) en el momento de la concesión esté indeterminado.

En virtud del primer caso estaríamos ante una situación sometida a alguna condición a priori para determinar los beneficiarios del plan de opciones sobre acciones. La empresa reconocerá inmediatamente los servicios recibidos, junto con el incremento del patrimonio neto, en la fecha de la concesión del plan (NIIF 2, 2005: párrafo 14), puesto que los servicios que están retribuyendo ya han sido prestados so pena que exista alguna prueba en contra, según establece la propia norma.

En el segundo caso, se presume "que los servicios se van a prestar por la otra parte, como contrapartida de esos instrumentos que recibirá en el futuro, durante el periodo de irrevocabilidad (o consolidación) de la concesión a lo largo del cual esos derechos se convierten en irrevocables" (NIIF 2, 2005: párrafo 15). Por ello, los servicios prestados se contabilizarán durante el periodo en que los derechos se conviertan en irrevocables.

De acuerdo con el tercer caso, si a un empleado se le conceden opciones sobre acciones en virtud a la condición de que alcance un determinado nivel de rentabilidad, éste debe permanecer en la empresa hasta que dicho nivel se logre. En esta situación, el periodo de consolidación no se puede conocer a priori porque éste va a variar dependiendo de cuándo se alcance ese nivel de rendimiento. En estas 
situaciones, la normativa establece que se presumirá que los servicios que prestará el empleado, como contrapartida de las opciones sobre acciones, se recibirán en el futuro a lo largo del periodo esperado de consolidación del derecho.

En estos casos, el periodo esperado se estimará basándose en el desenlace más probable de la condición impuesta de rendimiento, considerando los criterios siguientes (NIIF 2, 2005: párrafo 15b):

- Si el rendimiento se midiese en función de una condición relativa al mercado (por ejemplo, la cotización de las acciones de la empresa, la revalorización de las acciones con respecto a la experimentada por un determinado índice, etc.), la estimación de la duración del periodo esperado será coherente con las hipótesis empleadas para estimar el valor razonable de las opciones emitidas, lo cual no podrá ser revisado posteriormente.

- Si la condición de rendimiento no estuviese sujeta a condiciones de mercado (v.g.: objetivos de ventas o de resultados), la empresa revisará su estimación acerca de la duración del periodo de consolidación de los derechos, si fuera necesario, y siempre que la información posterior indicara que la duración del periodo de cumplimiento de la condición difiere de la prevista.

\section{Conclusiones}

La normativa internacional en materia contable regula que, cuando se produce un plan retributivo mediante la entrega de opciones sobre acciones a los empleados, el criterio de valoración sea el valor razonable de los instrumentos de patrimonio otorgados. Dicho valor debe ser calculado en el momento en que se produce la concesión de las citadas opciones, por lo que no será posible utilizar otra fecha porque cualquiera de ellas es posterior al momento en que la empresa incurre en el gasto como consecuencia de la aprobación de la entrega de las opciones.

Sin embargo, la normativa internacional no contempla la técnica de valoración concreta utilizable para llevar a cabo el cálculo del valor razonable de las opciones con el objetivo de no obstaculizar el desarrollo de técnicas de medición adecuadas, todavía inexistentes. Esta ausencia de definición de un modelo concreto plantea dudas acerca de si esta nueva norma conseguirá alcanzar uno de los objetivos esenciales de la contabilidad, la comparabilidad de los estados financieros. 
De los modelos más conocidos y utilizados en los mercados financieros para llevar a cabo la valoración de las opciones sobre acciones, consideramos que el modelo binomial permitirá obtener una mejor estimación de dicha valoración que la fórmula de Black-Scholes porque aquél introduce una mayor flexibilidad a la hora de recoger las diferentes variables (distintos valores de la tasa de interés y volatilidad para cada periodo) y las características propias de dichas opciones sobre acciones entregadas a los empleados.

De las variables por contemplar para la valoración de las opciones (precio de ejercicio, vida de la opción, precio del activo subyacente, volatilidad, dividendos y tasa de interés libre de riesgo), independientemente del modelo que se utilice, destaca la volatilidad (posible rango de variaciones de los precios del subyacente); ésta constituye el factor clave para la valoración de las opciones dado que es la única variable que no se conoce y que no se puede determinar a través del uso de ningún modelo o fórmula. La importancia de esta variable se pone de manifiesto en la propia normativa al dedicar especial atención a cómo debe determinarse su valor. Por ello, existe el riesgo de que las empresas utilicen las estimaciones de estas variables, especialmente de la volatilidad, con la finalidad de manipular el cálculo del valor de las opciones. 


\section{Referencias}

Bettis, J. C. , J. M. BizjaK y M. L. Lemmon (2003). "The cost of Employee Stock Options". http://ssrn.com/abstract=376440 [Fecha de consulta: 20/11/2008].

Black, F. y M. Scholes (1973). "The pricing of options and corporate liabilities". Journal of Political Economy, 81 (3): 637-659.

Bodie, Z., R. S. Kaplan y R. C. Merton (2003). "Las opciones sobre acciones son un gasto". Harvard Deusto Business Review, 115: 85-94.

Borrego Rodríguez, A. y P. García Estévez (2002). Productos financieros: sus mercados, valoración y estrategias de inversión. Madrid: Pearson Educación, 366. pp.

Boyle, P. P. (1977). “Options: Montecarlo approach”. Journal of Financial Economics, 4 (may): 323-338.

(1986). "Option valuation using a three-jump process". International Option Journal, 3: 7-12.

Brennan, M. J. y E. S. Schwartz (1977). "The valuation of American put options". Journal of Finance, 34 (may): 449-462.

(1978). "Finite difference methods and jump processes arising in the pricing of contingent claims: a synthesis". Journal of Financial and Quantitative Analysis, 13: 462-474.

Bulow, J. I. y J. B. Shoven (2005). “Accounting for stock options”. Research Paper Series Paper No.1848(R1) http://ssrn.com/abstract=521882 [Fecha de consulta: $2 / 6 / 2008]$.

CARPENTER, J.N. (1998). "The exercise and valuation of executive stock options". Journal of Financial Economics, 48 (2): 127-158.

CARR, P. y V. Linetsky (2000). "The valuation of executive stock options in an intensive-based framework". European Finance Review, 5 (3): 211-230. 
Córdoba Bueno, M. (2001). Guía de las stock options. Bilbao:Ediciones Deusto., $319 \mathrm{pp}$.

Cox, J., S. Ross y M. Rubinstein (1979). "Option pricing: A simplified approach". Journal of Financial Economics, 7: 229-264.

Díez De Castro, L. y J. Mascareñas Pérez-IÑIgo (1991). Ingeniería financiera: la gestión en los mercados financieros internacionales. Madrid: McGrawHill, $464 \mathrm{pp}$.

Financial Accounting Standard Board (Fasb) (2004). Statement of Financial Accounting Standards No. 123 (revised 2004): Share-Based Payment. Norwalk, Connecticut.

FinNerTy, T. D. (2005). "Extending the Black-Scholes-Merton model to value Employee Stock Options”. Journal of Applied Finance, 15 (2): 25-54.

HARTER, C. I. y T. HARIKUMAR (2002). "Accounting for option-based compensation: the economic cost approach". Journal of Business \& Accounting, 29 (7-8): 1007-1022.

Hemmer, T., S. Matsunaga y T. Shevlin (1994). (1996): “The influence of risk diversification on the early exercise of employee stock options by executive officers". Journal of Accounting and Economics, 21: 45-68.

Huddart, S. (1994). "Employee Stock Options". Journal of Accounting and Economics, 18 (2): 207-231.

Hull, J. y A. White (2004). "How to value Employee Stock Options". Financial Analysts Journal, 60 (1): 114-119.

Kulatilaka, N. y A. J. Marcus (1994). "Valuing Employee Stock Options". Financial Analysts Journal, 50 (6): 46-56.

Lambert, R.A., D. F. Larcker y R. E. Verrecchia (1991). "Portfolio consideration in valuing executive compensation". Journal of Accounting Research, 29 (1): 129-149. 
LAmothe Fernández, P. (1993). Opciones financieras: un enfoque fundamental. Madrid: McGraw-Hill, 322. pp.

Maris, B.A., J. Maris y T. T. YANG (2003). "The effect of exercise date uncertainty on Employee Stock Option value". Journal of Business Finance \& Accounting, 30 (5-6): 669-697.

Merton, R. C. (1973). “Theory of rational option pricing”. Bell Journal of Economics and Management Science, 4: 141-183.

Mock, S. (2005). "Accounting for Stock-based Compensation". European Business Law Review, 16 (2): 359-378.

NiSKANEN, W.A. (2004). "FASB is still wrong about stock options". http://www. cato.org/dailys/05-14-04.html [Fecha de consulta: 20/5/2008].

Raupach, P. (2003). "The valuation of Employee stock options - How good is the standard" http://wini-uni.com [Fecha de consulta: 15/6/2008].

Reglamento (CE) No 211/2005, de 4 de febrero, que modifica el Reglamento (CE) $\mathrm{n}^{\circ} 1725 / 2003$, en lo que respecta a las NIIF 1 y 2 y a las NIC, números 12 , 16, 19, 32, 33, 38 y 39 (Diario Oficial L 41 de 11/2/2005).

Rubinstein, M. (1994). "On the accounting valuation of Employee Stock Options". http://www.findarticles.com [Fecha de consulta: 20/11/2008].

StraWSER, J. (1993). "Accounting for stock-based compensation: the FASB's proposal". http://nysscpa.org/cpajournal/ old/14522920.htm [Fecha de consulta: $1 / 5 / 2008]$.

Templin, B. A. (2005). "Expensing isn't the only option: alternatives to the FASB's stock option expensing proposal". Journal of Corporation Law, 30 (2): 357-404. 


\section{Fórmula de Black-Scholes}

El modelo de Black-Scholes se basa en las siguientes hipótesis: a) el mercado funciona sin fricciones, es decir, no existen costos de transacción, de información ni impuestos y los activos son perfectamente divisibles; b) la transacción tiene lugar de forma continua y existe plena capacidad para realizar compras y ventas en descubierto ("a crédito") sin restricciones ni costos especiales; c) los agentes pueden prestar y endeudarse a una misma tasa " $r$ ", la tasa de interés a corto plazo expresado en forma de tasa instantánea y supuesto conocido y constante en el horizonte de valoración de las opciones; d) las opciones son europeas y el subyacente no paga dividendos en el horizonte de valoración; y e) el precio del subyacente sigue un proceso continuo estocástico de evolución de Gauss-Wiener. Por lo tanto, una cuestión fundamental para poder aplicar el modelo Black-Scholes y algunas de sus extensiones es que el rendimiento instantáneo aproxime su distribución a una distribución normal.

La fórmula por aplicar para llevar a cabo el cálculo del valor de una opción es la siguiente:

$$
\begin{gathered}
C=S N\left(d_{1}\right)-E e^{-r t} N\left(d_{2}\right) \\
d_{1}=\frac{\operatorname{Ln}\left(\frac{S}{E}\right)+\left(r+\frac{1}{2} \vartheta^{2}\right) t}{\vartheta \sqrt{t}} \\
d_{2}=d_{1}-\vartheta \sqrt{t}
\end{gathered}
$$

donde:

$\mathrm{S}=$ Precio del activo subyacente en el momento de la valoración; $\mathrm{E}=$ Precio de ejercicio; $r=$ Tasa de interés en tiempo continuo $[r=\operatorname{Ln}(1+i)] ; t=$ Plazo de ejercicio en años; $\vartheta=$ Volatilidad del precio del subyacente en términos anuales; e = Base de logaritmos neperianos; $\mathrm{N}$ (i) = Valor de la función de distribución normal para i. 


\section{Modelo binomial}

El modelo binomial se basa en las siguientes hipótesis: a) eficiencia y profundidad en los mercados; b) ausencia de costos de transacción; c) es posible comprar y vender en descubierto, sin límite; d) los activos son perfectamente divisibles; e) se puede prestar y tomar prestado a la misma tasa de interés; f) todas las transacciones se pueden realizar de forma simultánea; g) el precio del subyacente evoluciona según un proceso binomial multiplicativo.

Evolución del subyacente:

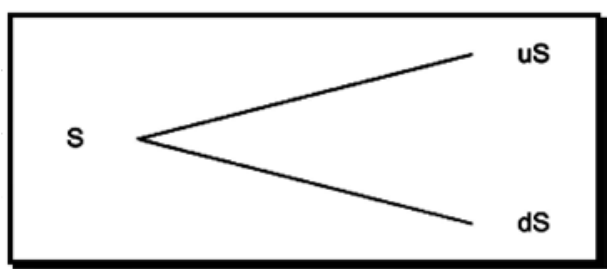

$\mathrm{u}=$ movimiento multiplicativo al alza del precio del subyacente en un periodo (probabilidad asociada de $\mathrm{q}$ ) $\mathrm{d}=$ movimiento multiplicativo a la baja del precio del subyacente en un periodo (probabilidad asociada de (1- q)). Así, la evolución de la opción:

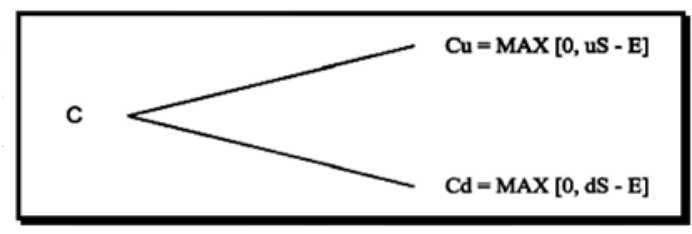

Por tanto: $\quad C=\frac{1}{\hat{r}}[p C u+(1-p) C d] ;$ donde: $p=\frac{\hat{r}-d}{u-d} ; \hat{r}=\left(1^{\prime}+r_{f}\right)$, siendo $r_{f}=$ Rentabilidad del activo libre de riesgo para un periodo 
Para "n" periodos, el precio del subyacente evolucionará de la siguiente forma:

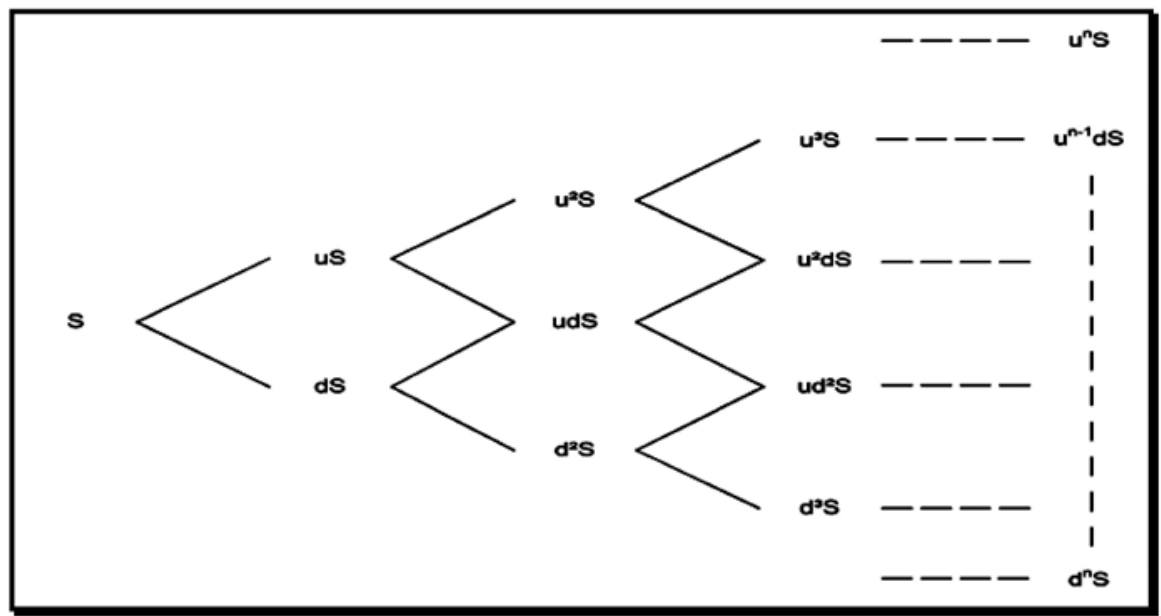

Por su parte, el valor de la opción evolucionará de la siguiente forma:

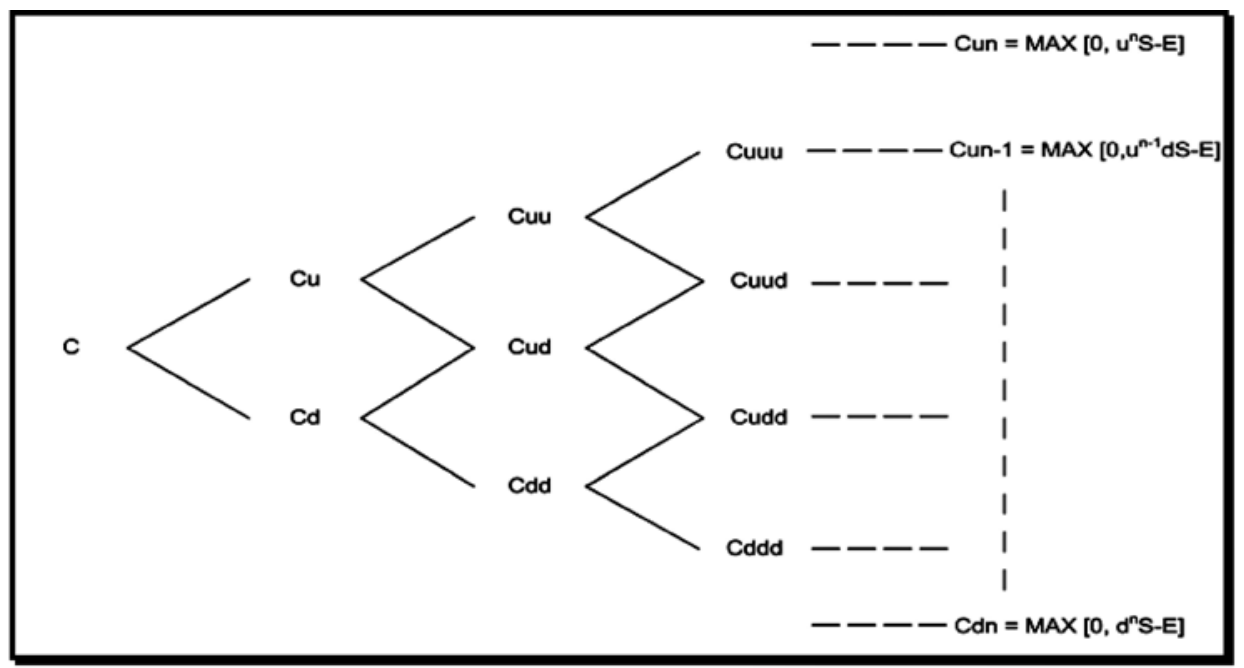


La valoración de la opción se obtendrá aplicando la fórmula siguiente, válida para "n" periodos:

$$
C=\frac{1}{\hat{r}^{n}}\left\{\sum_{j=0}^{n}\left(\frac{n !}{j !(n-j) !}\right) p^{j}(1-p)^{n-J} \operatorname{MAX}\left[0, u^{j} d^{n-J} S-E\right]\right\}
$$

(A) 